\title{
Enhanced self-efficacy after a self- management programme in pituitary disease: a randomized controlled trial
}

\author{
Cornelie D Andela', Han Repping-Wuts², Nike M M L Stikkelbroeck², \\ Mathilde C Pronk', Jitske Tiemensma ${ }^{3}$, Ad R Hermus², Adrian A Kaptein ${ }^{4}$, \\ Alberto M Pereira', Noelle G A Kamminga ${ }^{5}$ and Nienke R Biermasz ${ }^{1}$
}

1Division of Endocrinology, Department of Medicine, and Centre for Endocrine Tumors (CETL), Leiden University Medical Centre, Leiden, The Netherlands, ${ }^{2}$ Division of Endocrinology, Department of Internal Medicine, Radboud University Medical Centre, Nijmegen, The Netherlands, ${ }^{3}$ Psychological Science, University of California, Merced, CA, USA, ${ }^{4}$ Department of Medical Psychology, Leiden University Medical Centre, Leiden, The Netherlands, and ${ }^{5}$ Department of Psychiatry and Medical Psychology, Maastricht University Medical Centre, Maastricht, The Netherlands

\author{
Correspondence \\ should be addressed \\ to C D Andela \\ Email \\ c.d.andela@lumc.nl
}

\begin{abstract}
Context: Patients with pituitary disease report impairments in Quality of Life (QoL) despite optimal biomedical care. Until now, the effects of a self-management intervention (SMI) addressing psychological and social issues for these patients and their partners have not been studied.

Objective: To examine the effects of a SMI i.e. Patient and Partner Education Programme for Pituitary disease (PPEPPituitary).

Design and subjects: A multicentre randomized controlled trial included 174 patients with pituitary disease, and 63 partners were allocated to either PPEP-Pituitary or a control group. PPEP-Pituitary included eight weekly sessions (90 min). Self-efficacy, bother and needs for support, illness perceptions, coping and QoL were assessed before the intervention (T0), directly after (T1) and after six months (T2). Mood was assessed before and after each session. Results: Patients in PPEP-Pituitary reported improved mood after each session (except for session 1). In partners, mood only improved after the last three sessions. Patients reported higher self-efficacy at T1 $(P=0.016)$ which persisted up to T2 $(P=0.033)$, and less bother by mood problems directly after PPEP-Pituitary $(P=0.01)$, but more bother after six months $(P=0.001)$, although this increase was not different from baseline $(P=0.346)$. Partners in PPEP-Pituitary reported more vitality $(P=0.008)$ which persisted up to T2 $(P=0.034)$. At T2, partners also reported less anxiety and depressive symptoms $(P \leq 0.014)$.

Conclusion: This first study evaluating the effects of a SMI targeting psychosocial issues in patients with pituitary disease and their partners demonstrated promising positive results. Future research should focus on the refinement and implementation of this SMI into clinical practice.
\end{abstract}

\section{Introduction}

Patients with long-term biochemical remission of pituitary disease report impairments in Quality of Life (QoL) (1). Until now, little attention has been paid to interventions aiming at improving psychosocial aspects of QoL (2). The need for a psychosocial intervention in patients with pituitary disease was supported by results of recent focus group conversations reporting unmet needs regarding psychosocial care. Other reported issues in these focus groups were fatigue, increased sensitivity to stress, anxiety, depressive symptoms, difficulties
() 2017 European Society of Endocrinology Printed in Great Britain
European Journal of Endocrinology (2017) 177, 59-72 
communicating about the disease and a reduced social network (3). In addition, a focus group study in partners of patients with pituitary disease reported that partners sometimes became annoyed by the tiredness and mood swings of their ill partner. Some partners felt they had to take on extra responsibilities at home (e.g., taking care of the children). They were aware of the negative consequences of the disease on their family, but they felt unable to cope emotionally or physically (4).

For patients with other chronic somatic diseases, psychosocial interventions, i.e., self-management interventions (SMIs), have been developed aiming to improve well-being of patients (5). Self-management is defined by Barlow et al. as 'the individual's ability to manage the symptoms, treatment, physical and psychosocial consequences and life style changes inherent to living with a chronic condition. Efficacious self-management encompasses the ability to monitor one's condition and to affect the cognitive, behavioural and emotional responses necessary to maintain a satisfactory QoL' (6). SMIs in several chronic conditions (e.g., asthma, diabetes and arthritis) have demonstrated a positive effect on well-being of patients (6). Martire et al. (2010) demonstrated that couple-oriented interventions were more efficacious than psychosocial interventions that only included the patient or usual care (7). Although SMIs for any chronic disease may be based on general theoretical constructs, the composition and focus of the SMI depends on the type of disease and self-management aims, e.g., focus on the prevention of exacerbations in asthma, or focus on lifestyle habits in diabetes (8).

There are only a few studies evaluating the effect of a SMI in patients with neuroendocrine disease. MartinezMomblan et al. evaluated the effects of a 9-month educational nursing programme (5 visits) for patients with Cushing's syndrome in a randomized controlled trial $(n=61)$. This educational programme included knowledge on Cushing's syndrome, comorbidities, treatment, general management and autonomy in healthy lifestyles. Patients who followed this educational programme reported better disease-specific QoL, reduced pain, improved physical activity and a healthier lifestyle, compared to controls (9). Furthermore, Haugland et al. evaluated a 26-week educational programme in patients undergoing medical treatment for a neuroendocrine tumour in the gastrointestinal tract $(n=37)$, and demonstrated improvement in physical components of QoL, reduced stress and increased self-efficacy (10). These available SMIs focus primarily on education about Cushing's disease and its treatment and management (9) or education in patients with a neuroendocrine tumour in the gastrointestinal tract (10). Currently, a SMI for patients with pituitary disease and their partners addressing the psychosocial consequences and management of these consequences of the disease is lacking.

Considering the patient- and partner-reported need for psychosocial care in pituitary disease, and the current lack of a SMI addressing psychological and social issues in these patients and their partners, the aim of the present study was to evaluate the effects of such a SMI in a randomized controlled trial in a large cohort of patients with pituitary disease and their partners.

\section{Participants and methods}

\section{Design}

This multicentre two-arm randomized controlled trial was initiated by researchers at the Department of Medicine of the Leiden University Medical Centre (LUMC). Patients were randomized for the SMI or the control group; 1:1 randomization was performed by the first author (CDA). Partners of patients who agreed to participate $(n=63)$ were allocated to the same condition as their ill partner.

For ethical reasons, patients and partners who were randomized to the control group were also offered the SMI after the last measurement. The medical ethical committee of the LUMC approved the study, and written informed consent was obtained from all participants.

\section{Participants}

The recruitment was coordinated by the outpatient departments of Medicine of the LUMC and the Radboud University Medical Centre (Radboudumc). Exclusion criteria were: $<18$ or $>75$ years of age since older patients might have more comorbidity, current psychological treatment, current intensive medical treatment (e.g., radiotherapy, recovery from surgery) and psychiatric illness. A total number of 931 patients (and their partner when applicable) were informed about the study and were invited to participate (i.e., 462 from the LUMC; 469 from the Radboudumc). Reasons for not participating in the study were not speaking Dutch, not feeling comfortable talking in a group, too time-consuming, burden too large (physically and/or mentally), not able to come due to other obligations (e.g. work, staying abroad, pregnancy, surgery), long travel distance, not perceiving problems and no need for support (anymore), because patients already receive psychological counselling or previously received 
it, or learned to cope with their illness by themselves. One hundred and eight patients (LUMC) and 80 patients (Radboudumc) agreed to participate. From the initial 188 incorporated patients, fourteen patients (7\%) did not fill out the questionnaires. Therefore, a total number of 174 patients were included (Fig. 1).

\section{Development of the SMI}

The SMI was based on the standardized Patient (and Partner) Education Programme initially developed for Parkinson's disease (PEPP), and evaluated in seven European countries $(11,12)$ including the Netherlands $(13,14,15)$, and is currently operational in patient care. The programme was then adapted for Huntington's disease (PEP-HD) (16) and was further developed and clinically tested in patients with chronic disease with psychiatric comorbidity (17). Since the self-management techniques seemed to be generally applicable, the programme has recently been developed for patients with chronic disease in general (PPEP4ALL) (18). PPEP4ALL addresses psychological and social issues related to all chronic disease and uses techniques from cognitive behavioural therapy such as cognitive restructuring, systematic relaxation training, situational behavioural analyses and training in social skills.

In order to assess whether PEPP was also suitable for patients with pituitary disease, focus group conversations in patients with pituitary disease were performed (3). The focus group guided us in laying the priorities and preferred options (e.g. fatigue, cognitive complaints and problems with sexuality) within the PPEP4ALL. Based on these results, we hypothesized that PEPP/PPEP4ALL (Fig. 2) would also be of relevance for patients with pituitary disease and their partners (when applicable). Then, we pilot tested it in 28 patients and 6 partners. Patients and partners reacted positively to the programme. Therefore, we decided to evaluate PPEP4ALL with the preferred options: fatigue, cognitive complaints and problems with sexuality. It was not necessary to drop any of the other components of PPEP4ALL, and considering the patient group, we named it the 'Patient

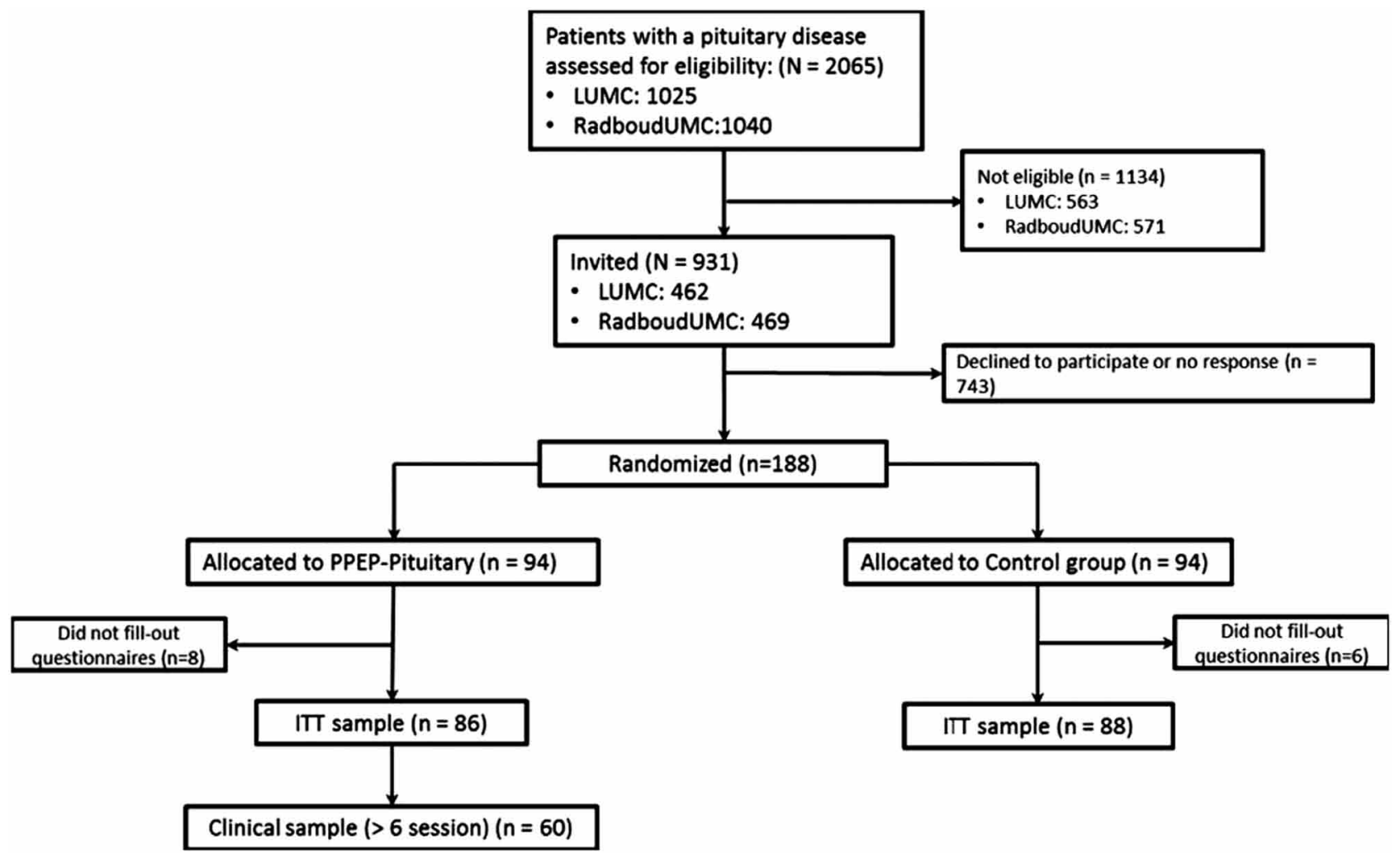

Figure 1

Flow chart of patients. 


\section{Session 1}

Information

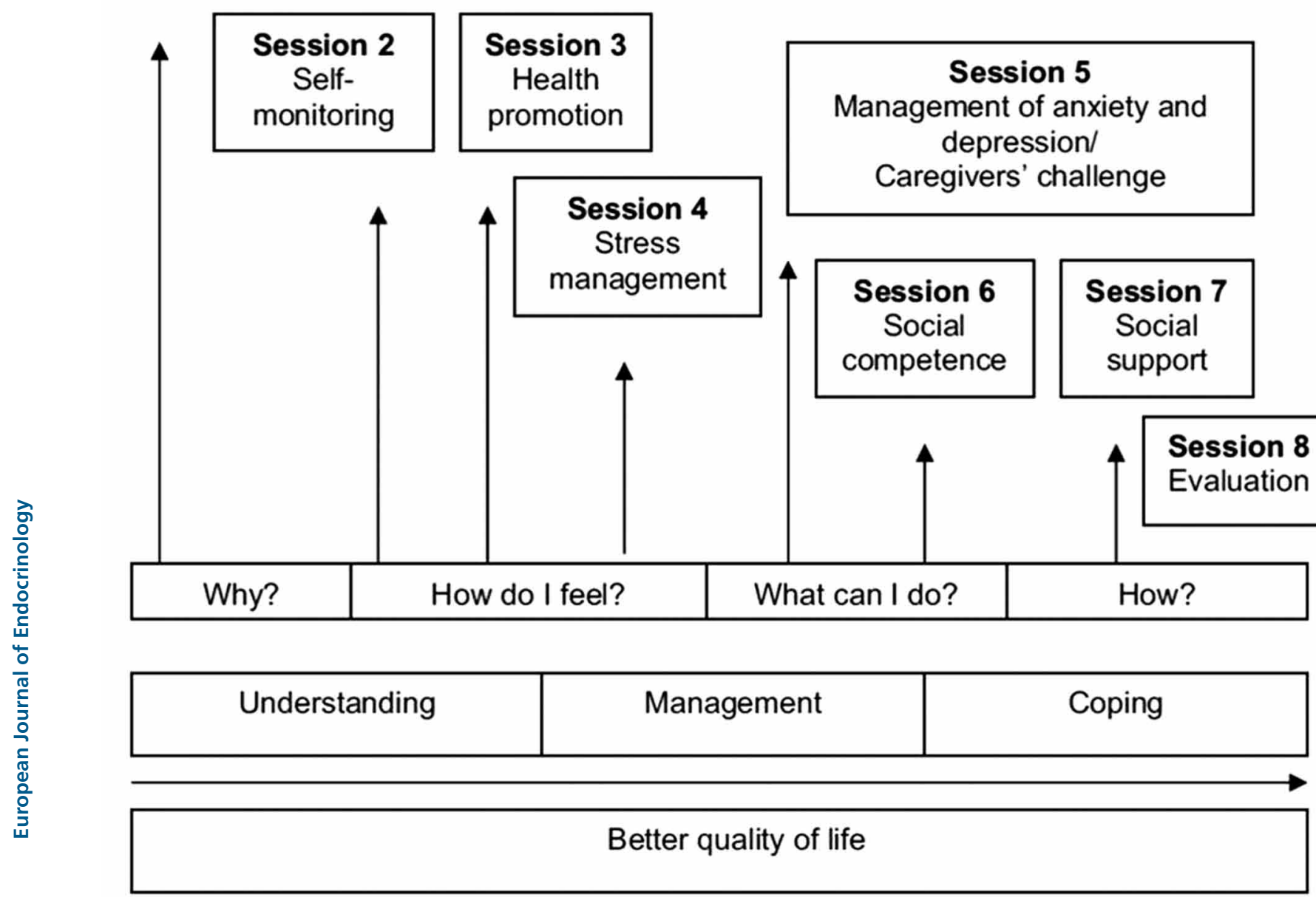

Figure 2

The themes and aims of the PPEP-Pituitary.

and Partner Education Programme for Pituitary disease (PPEP-Pituitary)'.

\section{PPEP-Pituitary}

PPEP-Pituitary includes self-management components of potential relevance for pituitary disease, i.e., fatigue management, stress management, dealing with anxiety and depression, and communication training (Supplementary Data 1, see section on supplementary data given at the end of this article). The programme consisted of eight weekly sessions of 90 min moderated by psychologists and medical social workers. Patients and partners participated separately and from their own perspective, in groups of 5-7 participants at the LUMC or at the Radboudumc. The same one or two trainers guided each group for 8 weeks (CDA, SM, RM, NF, MP-D, RG, $\mathrm{JL}, \mathrm{MS}$ and $\mathrm{MV})$. All trainers were trained in/experienced with the PPEP/PPEP4ALL, and followed a one-day training to get familiar with the disease-specific focus on pituitary disease.

\section{Procedure}

All included participants were asked to fill out questionnaires prior to the programme (T0). Next, participants in PPEP-Pituitary followed the 8-week SMI, while the participants in the control group were invited 
for a single (optional) information meeting in week 4 or 5 . For the formation of the patient groups, groups were stratified by disease in 3 groups i.e., 1) Cushing's disease (CD), 2) acromegaly or 3) prolactinoma/nonfunctioning pituitary adenoma (NFA)/FSH-adenoma/ craniopharyngioma/hypopituitarism due to other causes. Partners in PPEP-Pituitary were not stratified by pituitary disease of their partner. Participants were asked to fill out the questionnaires again after the 8-week intervention (T1) and 6 months later (T2). Demographic characteristics (i.e., age, gender, marital status and education) and medication use were assessed by a self-report. Clinical characteristics of patients (e.g., type of pituitary disease and duration of follow-up) were derived from medical records.

\section{Measures}

For an overview of the used measures, see Table 1.

Based on the preference of participants, questionnaires were sent by email (online survey) or by regular mail to increase the response rate. One hundred and nine patients and 53 partners completed the questionnaires online, and 65 patients and 10 partners by postal survey. Previous research demonstrated that paper-and-pencil and online surveys do not lead to different results (19). Partners completed the same questionnaires except the LBNQPituitary, the EQ-5D, the IPA and the disease-specific QoL questionnaires (i.e., AcroQoL, CushingQoL). In addition, patients and partners in the PPEP-Pituitary group were asked to fill out an evaluation form about PPEP-Pituitary (Supplementary Data 2).

\section{Statistical analyses}

Data were analysed using PASW Statistics version 20.0.0 (SPSS Inc.). To check the normality of data, the Kolmogorov-Smirnov test was used. Demographic characteristics and the baseline scores (Supplementary Data 3) were compared using independent sample t-test and chi-square test when data were normally distributed and by using Mann-Whitney $U$ test and Fisher's exact when data were not normally distributed. To compare pre- and post-session mood ratings, paired sample t-tests were used. A linear mixed model with random participant effect, and fixed time and group effects, as well as group by time interactions measured the effects of the programme. The linear mixed model enables accommodating missing data points (38), and corrects for potential baseline differences. The effects of the programme were evaluated following intention to treat (ITT) principles, including all participants. Although ITT analysis is the golden standard for analysing an RCT, it is also considered conservative (39) since not all participants in PPEP-Pituitary attended all sessions. Therefore, the post hoc analyses comprised the clinical sample analyses including only the patients that attended at least six sessions, since this is the minimum amount of sessions to consider that someone completed PPEP-Pituitary, and since this situation will be more similar to the clinical situation. This analysis was performed using the same linear mixed model. The data from the evaluation were analysed descriptively. Due to the explorative nature of this study, the level of significance was set at $P<0.05$. However, to take into account the effect of multiple testing, a Bonferroni correction was applied, and a level of significance of $P<0.005$ was also used.

\section{Results}

\section{Baseline characteristics}

Of the 188 patients incorporated, 94 patients were allocated to PPEP-Pituitary and 94 patients to the control group. Fourteen patients (8 in PPEP-Pituitary and 6 in the control group) did not complete any of the questionnaires and were not included in the ITT analysis. Therefore, a final number of 174 patients were included in the ITT analysis (PPEP-Pituitary: $n=86$ and control group: $n=88$ ). Seventy per cent of the patients $(n=60)$ in PPEP-Pituitary attended at least 6 sessions i.e., the clinical sample.

From this clinical sample, 12 patients (20\%) attended 6 sessions, 24 patients (40\%) attended 7 sessions and another 24 patients (40\%) attended all 8 sessions.

From the patients in the control group, 42 patients (48\%) attended the optional information meeting. Furthermore, $70 \%$ of the patients were married or in a relationship ( $n=122)$, and 63 partners $(52 \%)$ were willing to participate. Twenty-five partners were in the PPEPPituitary group, and 38 partners were in the control group. From the partners in PPEP-Pituitary, 52\% $(n=13)$ attended at least 6 sessions (i.e., the clinical sample). From the partners in the control group, 16 (42\%) attended the optional information meeting (Table 2).

\section{Mood changes after each PPEP-Pituitary session}

Patient reported mood improved significantly after each session (all $P<.001$ ), except for session 1 . Partners' mood 
Table 1 Measures.

\begin{tabular}{|c|c|c|}
\hline Questionnaire & Outcome & Subscales \\
\hline $\begin{array}{l}\text { Visual analogue } \\
\text { scale-Mood } \\
\text { (VAS-Mood) }\end{array}$ & Mood & 1 \\
\hline $\begin{array}{c}\text { General self-efficacy } \\
\text { scale (GSE) }(20,21)\end{array}$ & Self-efficacy & 1 \\
\hline
\end{tabular}
sexual functioning,
Leiden bother and needs questionnaire for pituitary diseases (LBNQPituitary) (22)

Brief Illness perception questionnaire (B-IPQ) (23)

\section{the Utrecht coping} list (UCL) (24)

\section{Bother and need for support \\ Illness perceptions}

\section{Coping} strategies

$\begin{array}{ll}\text { Impact on } & \text { Participation } \\ \text { participation and } & \text { and } \\ \text { autonomy (IPA) (25) } & \text { autonomy }\end{array}$

EuroQoL-5D (EQ-5D) QoL (26)

Short form 36 (SF-36) QoL $(27,28)$
5: Mood, Negative illness perceptions, Issues in Physical and cognitive complaints, Issues in social functioning

8: Consequences, Personal control, Timeline, Treatment control, Identity, Concern, Coherence, Emotional response 7: Active coping, Seeking distraction, Avoiding, Seeking social support, Passive coping,

Expressing emotions, Fostering reassuring thoughts

5: family role, autonomy outdoors, autonomy indoors, social life and relationships, work and education

5: mobility, self-care, usual activities, pain/ discomfort, and anxiety/ depression

9: physical functioning, social functioning, role limitation (physical), role limitation (emotional), mental health, vitality, pain, general health perception, general perception of change in health

\begin{tabular}{|c|c|}
\hline $\begin{array}{l}\text { Number of } \\
\text { items }\end{array}$ & Range \\
\hline 1 & $\begin{array}{l}0-100,0=\text { extremely } \\
\text { bad mood to } \\
100=\text { extremely } \\
\text { good mood }\end{array}$ \\
\hline 10 & $\begin{array}{l}10-40, \uparrow \text { scores } \\
\text { indicate } \uparrow \text { self- } \\
\text { efficacy }\end{array}$ \\
\hline
\end{tabular}

26

0-100, $\uparrow$ scores indicate $\uparrow$ bother and need for support

$0-10,0=$ not at all to $10=$ very much

Active coping (7-28) Seeking distraction (8-32), Avoiding (8-32), Seeking social support (6-24), Passive coping (7-28), Expressing emotions (3-12), Fostering reassuring thoughts (5-20). $\uparrow$ scores indicate $\uparrow$ more frequent performance of that coping

$32 \quad 0-4, \downarrow$ scores indicate $\uparrow$ participation and autonomy

$1-3, \uparrow$ scores indicate $\downarrow$ QoL

0-100, $\uparrow$ scores indicate $\uparrow Q \circ L$

\begin{tabular}{l}
$\begin{array}{c}\text { Measurement } \\
\text { time }\end{array}$ \\
\hline $\begin{array}{l}\text { Before and } \\
\text { after each } \\
\text { session }\end{array}$
\end{tabular}

T0, T1, T2

$\mathrm{T} 0, \mathrm{~T} 1, \mathrm{~T} 2$

T0, T1, T2

Participants

Patients and partners in PPEP-

Pituitary

Patients and partners in PPEP-

Pituitary and control group

Patients and partners in PPEP-

Pituitary and control group

Patients and partners in PPEP-

Pituitary and control group

T0, T1, T2

Patients and partners in PPEP-

Pituitary and control group

T0, T1, T2

Patients and partners in PPEP-

Pituitary and control group Patients and partners in PPEP-

Pituitary and control group Patients and partners in PPEP-

Pituitary and control group 
Table 1 Continued.

\begin{tabular}{|c|c|c|}
\hline Questionnaire & Outcome & Subscales \\
\hline $\begin{array}{l}\text { Multidimensional } \\
\text { fatigue inventory } \\
\text { (MFI-20) (29) }\end{array}$ & Fatigue & $\begin{array}{l}\text { 5: General fatigue, } \\
\text { Physical fatigue, } \\
\text { Reduced activity, } \\
\text { Reduced motivation, } \\
\text { Mental fatigue }\end{array}$ \\
\hline $\begin{array}{l}\text { Hospital anxiety and } \\
\text { depression scale } \\
\text { (HADS) }(30,31)\end{array}$ & $\begin{array}{l}\text { Anxiety and } \\
\text { depression }\end{array}$ & $\begin{array}{l}\text { 2: anxiety, depressive } \\
\text { symptoms }\end{array}$ \\
\hline AcroQoL $(32,33,34)$ & $\begin{array}{l}\text { Disease- } \\
\text { specific QoL }\end{array}$ & $\begin{array}{l}\text { 3: physical, psychological- } \\
\text { appearance, } \\
\text { psychological-personal } \\
\text { relations }\end{array}$ \\
\hline $\begin{array}{r}\text { CushingQoL } \\
(35,36,37)\end{array}$ & $\begin{array}{l}\text { Disease-spe- } \\
\text { cific QoL }\end{array}$ & $\begin{array}{l}\text { 2: Psychosocial issues, } \\
\text { physical problems }\end{array}$ \\
\hline
\end{tabular}

\begin{tabular}{|c|c|c|c|}
\hline $\begin{array}{l}\text { Number of } \\
\text { items }\end{array}$ & Range & $\begin{array}{c}\text { Measurement } \\
\text { time }\end{array}$ & Participants \\
\hline 20 & $\begin{array}{l}\text { 0-20, } \uparrow \text { scores } \\
\text { indicate } \uparrow \text { fatigue }\end{array}$ & T0, T1, T2 & $\begin{array}{l}\text { Patients and } \\
\text { partners in } \\
\text { PPEP- } \\
\text { Pituitary and } \\
\text { control } \\
\text { group }\end{array}$ \\
\hline 14 & $\begin{array}{l}\text { 0-21, } \uparrow \text { scores } \\
\text { indicate } \uparrow \text { anxiety/ } \\
\text { depressive } \\
\text { symptoms }\end{array}$ & $\mathrm{T} 0, \mathrm{~T} 1, \mathrm{~T} 2$ & $\begin{array}{l}\text { Patients and } \\
\text { partners in } \\
\text { PPEP- } \\
\text { Pituitary and } \\
\text { control } \\
\text { group }\end{array}$ \\
\hline 22 & $\begin{array}{l}\text { 0-100, } \downarrow \text { scores } \\
\text { indicate } \downarrow \text { QoL }\end{array}$ & $\mathrm{T} 0, \mathrm{~T} 1, \mathrm{~T} 2$ & $\begin{array}{l}\text { Patients with } \\
\text { acromegaly } \\
\text { in PPEP- } \\
\text { Pituitary and } \\
\text { control } \\
\text { group }\end{array}$ \\
\hline 12 & $\begin{array}{l}\text { 0-100, } \downarrow \text { scores } \\
\text { indicate } \downarrow \text { QoL }\end{array}$ & T0, T1, T2 & $\begin{array}{l}\text { Patients with } \\
\text { CD in } \\
\text { PPEP-Pitu- } \\
\text { itary and } \\
\text { control } \\
\text { group }\end{array}$ \\
\hline
\end{tabular}

improved only after sessions 6,7 and 8 (all $P \leq .030$ ) (Table 3).

\section{Effects of intervention: Intention to treat analysis}

Self-efficacy

For patients a significant interaction was found for selfefficacy (GSE) $(P=0.002)$, with PPEP-Pituitary reporting more self-efficacy compared to controls (difference 1.35, $P=0.016$ ) (T1 vs T0), which persisted up to the 6-month follow-up (difference 1.74, $P=0.033$ ) (T2 vs T0). No significant difference in self-efficacy was observed in partners (Table 4).

\section{Bother and needs for support}

An interaction was found for being bothered by mood problems (LBNQ-Pituitary) $(P=0.002)$ with PPEPPituitary, reporting to be less bothered by mood problems compared to controls (difference -6.27, $P=0.010$ ) (T1 vs T0). At T2 relative to T1, PPEP-Pituitary reported more bother by mood problems compared to controls (difference 8.71, $P=0.001$ ), but this increase at T2 was not significantly different from baseline (difference 2.44, $P=0.346$ ). Furthermore, an interaction was observed on the total score of the bothered by items of the LBNQPituitary $(P=0.028)$, with PPEP-Pituitary reporting more overall bother (total score) at $\mathrm{T} 2$ relative to $\mathrm{T} 1$ compared to controls (difference $4.58, P=0.008$ ), but this increase at T2 was also not significantly different from baseline (difference 2.20, $P=0.219$ ).

\section{IIIness perceptions}

No significant differences in illness perceptions (B-IPQ) were observed for patients over time.

For partners, an interaction was found for perceived treatment control $(P=0.025)$, with PPEP-Pituitary perceiving more treatment control compared to controls (difference 3.12, $P=0.008$ ) (T2 vs T1), but this increase at T2 was not significant from baseline (difference 1.43, $P=0.230$ )

\section{Coping}

No significant differences in coping styles (UCL) were found for patients and partners over time.

\section{Participation and autonomy}

No significant differences in participation and autonomy (IPA) were found for patients over time.

\section{Quality of life}

For patients, no significant differences were found for QoL (i.e., EQ-5D, SF-36, MFI-20, HADS, CushingQoL, AcroQoL). 
Table 2 Demographic variables of patients and partners.

\begin{tabular}{|c|c|c|c|c|c|c|}
\hline & \multicolumn{3}{|c|}{ Patients $(n=174)$} & \multicolumn{3}{|c|}{ Partners $(n=63)$} \\
\hline & $\begin{array}{l}\text { PPEP-Pituitary } \\
\text { group }(n=86)\end{array}$ & $\begin{array}{l}\text { Control group } \\
\quad(n=88)\end{array}$ & $P$ value & $\begin{array}{l}\text { PPEP-Pituitary } \\
\text { group }(n=25)\end{array}$ & $\begin{array}{c}\text { Control group } \\
\quad(n=38)\end{array}$ & $P$ value \\
\hline Gender (M/F) & $33 / 53$ & $31 / 57$ & 0.667 & $17 / 8$ & $18 / 20$ & 0.107 \\
\hline Age (years) & $52.7(11.9)$ & $53.4(12.7)$ & 0.600 & $55.7(10.6)$ & $58.9(9.9)$ & 0.298 \\
\hline Condition/condition of ill partner, $n(\%)$ & & & 0.866 & & & 0.835 \\
\hline Cushing's disease & $21(24 \%)$ & $19(22 \%)$ & & $7(28 \%)$ & $6(16 \%)$ & \\
\hline Acromegaly & $12(14 \%)$ & $10(11 \%)$ & & $5(20 \%)$ & $7(18 \%)$ & \\
\hline Prolactinoma & $18(21 \%)$ & $20(23 \%)$ & & $3(12 \%)$ & $9(24 \%)$ & \\
\hline NFA & $27(31 \%)$ & $30(34 \%)$ & & $8(32 \%)$ & $13(34 \%)$ & \\
\hline FSH-adenoma & $0(0 \%)$ & $1(1 \%)$ & & $0(0 \%)$ & $0(0 \%)$ & \\
\hline Craniopharyngioma & $5(6 \%)$ & $3(3 \%)$ & & $2(8 \%)$ & $0(0 \%)$ & \\
\hline Hypopituitarism due to other causes & $3(4 \%)$ & $5(6 \%)$ & & $0(0 \%)$ & $1(3 \%)$ & \\
\hline Education, $n(\%)$ & & & 0.219 & & & 0.238 \\
\hline Low & $20(23 \%)$ & $27(31 \%)$ & & $4(16 \%)$ & $13(34 \%)$ & \\
\hline Medium & $23(27 \%)$ & $29(33 \%)$ & & $11(44 \%)$ & $11(29 \%)$ & \\
\hline High & $42(49 \%)$ & $32(36 \%)$ & & $10(40 \%)$ & $14(37 \%)$ & \\
\hline Unknown & $1(1 \%)$ & $0(0 \%)$ & & $0(0 \%)$ & $0(0 \%)$ & \\
\hline Marital status, $n(\%)$ & & & 0.027 & & & NA \\
\hline Single & $16(19 \%)$ & $11(13 \%)$ & & $0(0 \%)$ & $0(0 \%)$ & \\
\hline Relationship/marriage & $55(64 \%)$ & $67(76 \%)$ & & $25(100 \%)$ & $38(100 \%)$ & \\
\hline Divorced & $8(9 \%)$ & $9(10 \%)$ & & $0(0 \%)$ & $0(0 \%)$ & \\
\hline Widow & $7(8 \%)$ & $0(0 \%)$ & & $0(0 \%)$ & $0(0 \%)$ & \\
\hline Unknown & $0(0 \%)$ & $1(1 \%)$ & & $0(0 \%)$ & $0(0 \%)$ & \\
\hline Pituitary surgery, $n(\%)$ & $60(70 \%)$ & $63(72 \%)$ & 0.792 & NA & NA & NA \\
\hline Radiotherapy, $n(\%)$ & $19(22 \%)$ & $21(24 \%)$ & 0.781 & NA & NA & NA \\
\hline Duration since diagnosis (years) & $11.7(10.8)$ & $13.0(13.5)$ & 0.884 & NA & NA & NA \\
\hline \multicolumn{7}{|l|}{ Hypopituitarism, $n(\%)$} \\
\hline АCTH & $40(47 \%)$ & $50(57 \%)$ & 0.174 & NA & NA & NA \\
\hline TSH & $42(49 \%)$ & $46(52 \%)$ & 0.650 & NA & NA & NA \\
\hline LH/FSH & $40(47 \%)$ & $41(47 \%)$ & 0.992 & NA & NA & NA \\
\hline $\mathrm{GH}$ & $36(42 \%)$ & $40(46 \%)$ & 0.633 & NA & NA & NA \\
\hline $\mathrm{ADH}$ & $6(7 \%)$ & $8(9 \%)$ & 0.608 & NA & NA & NA \\
\hline
\end{tabular}

For partners, an interaction was found for vitality (SF-36) $\quad(P=0.026)$, with PPEP-Pituitary reporting more vitality compared to controls (difference 14.03, $P=0.008$ ) (T1 vs T0), which persisted up to the 6-month follow-up (difference 15.45, $P=0.034$ ) (T2 vs T0). Furthermore, an interaction was found for anxiety (HADS) $(P=0.035)$, with PPEP-Pituitary reporting less anxiety at T2 relative to T0 (difference $-2.65, P=0.014$ ).
In addition, an interaction was found for depressive symptoms (HADS) $(P=0.012)$, with PPEP-Pituitary reported less depressive symptoms at $\mathrm{T} 2$ relative to T0 (difference $-3.47, P=0.003$ ), as well as at T2 relative to T1 (difference $-2.60, P=0.012$ ). Finally, an interaction was found for the HADS total score $(P=0.005)$, with PPEP-Pituitary reporting a lower total HADS score at T2 relative to T0 (difference $-6.51, P=0.002$ ), as well as at

Table 3 Pre- and post-session mood-VAS ratings (range 0-100) of patients and partners. Data are presented as mean (s.D.).

\begin{tabular}{|c|c|c|c|c|c|c|}
\hline \multirow[b]{2}{*}{ Session } & \multicolumn{3}{|c|}{ Patients $(n=54-70)$} & \multicolumn{3}{|c|}{ Partners $(n=10-15)$} \\
\hline & Before session & After session & $P$ value & Before session & After session & $P$ value \\
\hline 1 & $69.91(13.14)$ & 70.94 (12.09) & 0.384 & $71.15(10.24)$ & 71.92 (12.17) & 0.776 \\
\hline 2 & 68.03 (14.83) & 74.32 (12.27) & $<0.001 *$ & $72.00(9.02)$ & $73.13(10.46)$ & 0.687 \\
\hline 3 & $65.27(14.48)$ & $73.11(12.41)$ & $<0.001 *$ & $68.75(14.32)$ & $74.83(9.11)$ & 0.090 \\
\hline 4 & 68.96 (12.93) & 75.39 (10.32) & $<0.001 *$ & 70.58 (8.37) & 73.42 (7.83) & 0.055 \\
\hline 5 & $68.77(10.55)$ & 73.55 (11.94) & $<0.001 *$ & $73.60(8.51)$ & $73.40(7.90)$ & 0.920 \\
\hline 6 & $67.96(12.81)$ & $73.18(11.22)$ & $<0.001 *$ & $73.00(6.95)$ & $77.17(6.46)$ & $0.005 *$ \\
\hline 7 & $70.76(10.02)$ & $75.25(9.43)$ & $<0.001 *$ & $75.08(7.32)$ & $78.15(7.03)$ & $0.025 *$ \\
\hline 8 & $70.65(10.48)$ & $77.93(9.33)$ & $<0.001 *$ & $73.08(6.09)$ & $77.54(7.66)$ & $0.030 *$ \\
\hline
\end{tabular}


Table 4 Changes in the outcome measures in patients and partners (ITT sample).

\begin{tabular}{|c|c|c|c|c|c|c|c|c|}
\hline \multirow{2}{*}{ 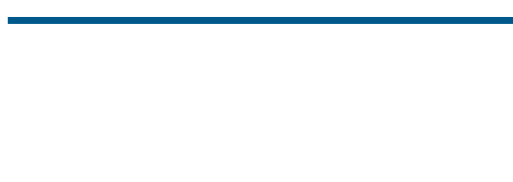 } & \multicolumn{3}{|c|}{ Patients $(n=174)$} & \multirow{2}{*}{$\begin{array}{c}P \text { value } \\
\text { group } \times \\
\text { time }\end{array}$} & \multicolumn{3}{|c|}{ Partners $(n=63)$} & \multirow{2}{*}{$\begin{array}{c}P \text { value } \\
\text { group } \times \\
\text { time }\end{array}$} \\
\hline & $\begin{array}{c}\triangle \text { PPEP- } \\
\text { control } \\
\text { T0-T1 }\end{array}$ & $\begin{array}{c}\triangle \text { PPEP- } \\
\text { control } \\
\text { T1-T2 }\end{array}$ & $\begin{array}{c}\triangle \text { PPEP- } \\
\text { control } \\
\text { T0-T2 }\end{array}$ & & $\begin{array}{c}\triangle \text { PPEP- } \\
\text { control } \\
\text { T0-T1 }\end{array}$ & $\begin{array}{c}\triangle \text { PPEP- } \\
\text { control } \\
\text { T1-T2 }\end{array}$ & $\begin{array}{l}\triangle \text { PPEP- } \\
\text { control } \\
\text { T0-T2 }\end{array}$ & \\
\hline \multicolumn{9}{|l|}{ LBNQ-Pituitary } \\
\hline \multicolumn{9}{|l|}{ Bother by } \\
\hline Mood problems & $-6.27 *$ & $8.71^{\#}$ & 2.44 & $0.002^{\#}$ & NA & NA & NA & NA \\
\hline Negative illness perceptions & -2.14 & 4.03 & 1.89 & 0.176 & NA & NA & NA & NA \\
\hline Issues in sexual functioning & 2.92 & 2.02 & 4.94 & 0.272 & NA & NA & NA & NA \\
\hline Physical and cognitive complaints & -0.77 & 4.66 & 3.89 & 0.127 & NA & NA & NA & NA \\
\hline Issues in social functioning & -3.06 & 1.54 & -1.52 & 0.347 & NA & NA & NA & NA \\
\hline Total score & -2.37 & $4.58^{*}$ & 2.20 & $0.028^{*}$ & NA & NA & NA & NA \\
\hline \multicolumn{9}{|l|}{ Need for support } \\
\hline Mood problems & -4.69 & 8.26 & 3.57 & 0.073 & NA & NA & NA & NA \\
\hline Negative illness perceptions & -1.92 & 6.33 & 4.41 & 0.131 & NA & NA & NA & NA \\
\hline Issues in sexual functioning & 4.09 & 2.03 & 6.12 & 0.309 & NA & NA & NA & NA \\
\hline Physical and cognitive complaints & 0.79 & 5.85 & 6.63 & 0.077 & NA & NA & NA & NA \\
\hline Issues in social functioning & -4.38 & 2.75 & -1.64 & 0.199 & NA & NA & NA & NA \\
\hline Total score & -1.65 & 5.17 & 3.53 & 0.078 & NA & NA & NA & NA \\
\hline \multicolumn{9}{|l|}{ EQ-5D } \\
\hline Mobility & -0.07 & 0.04 & -0.03 & 0.509 & NA & NA & NA & NA \\
\hline Self-care & 0.01 & 0.01 & 0.02 & 0.904 & NA & NA & NA & NA \\
\hline Daily activity & -0.15 & 0.05 & -0.10 & 0.167 & NA & NA & NA & NA \\
\hline Pain & -0.03 & -0.04 & -0.06 & 0.781 & NA & NA & NA & NA \\
\hline Mood & -0.06 & -0.05 & -0.10 & 0.408 & NA & NA & NA & NA \\
\hline VAS & -3.63 & 0.84 & -2.79 & 0.352 & NA & NA & NA & NA \\
\hline \multicolumn{9}{|l|}{ SF-36 } \\
\hline Physical functioning & 3.45 & -4.82 & -1.37 & 0.211 & 1.95 & 7.66 & 9.62 & 0.302 \\
\hline Social functioning & 2.08 & -1.92 & 0.17 & 0.722 & 3.04 & 14.71 & 17.75 & 0.084 \\
\hline Role limitations-Physical & 2.39 & -12.03 & -9.64 & 0.203 & -6.87 & 29.52 & 22.65 & 0.111 \\
\hline Role limitations-Emotional & -3.31 & 3.19 & -0.12 & 0.831 & -15.99 & 30.92 & 14.93 & 0.156 \\
\hline Mental Health & 1.63 & -2.18 & -0.55 & 0.480 & -6.26 & 11.61 & 5.35 & 0.330 \\
\hline Vitality & 3.14 & -3.43 & -0.29 & 0.284 & $14.03 *$ & 1.42 & $15.45^{*}$ & $0.026 *$ \\
\hline Pain & -2.62 & -0.79 & -3.40 & 0.505 & -4.42 & 9.57 & 5.15 & 0.281 \\
\hline General Health & -0.62 & 3.77 & 3.14 & 0.179 & 2.82 & -2.66 & 0.17 & 0.729 \\
\hline Health change & 0.03 & -7.79 & -7.50 & 0.234 & 12.72 & 8.74 & 21.46 & 0.083 \\
\hline \multicolumn{9}{|l|}{ MFI-20 } \\
\hline General fatigue & -0.37 & 0.40 & 0.03 & 0.441 & 1.18 & -0.58 & 0.61 & 0.215 \\
\hline Physical fatigue & 0.20 & -0.42 & -0.22 & 0.389 & -0.13 & -0.10 & -0.23 & 0.932 \\
\hline Reduced activity & -0.20 & 0.27 & 0.07 & 0.731 & 0.44 & -0.70 & -0.26 & 0.700 \\
\hline Reduced motivation & 0.07 & 0.02 & 0.10 & 0.961 & -0.40 & -0.48 & -0.88 & 0.373 \\
\hline Mental fatigue & 0.37 & 1.44 & 1.82 & 0.107 & -0.16 & -0.88 & -1.04 & 0.152 \\
\hline \multicolumn{9}{|l|}{ HADS } \\
\hline Anxiety & -0.09 & 0.07 & -0.02 & 0.976 & -0.91 & -1.74 & $-2.65^{*}$ & $0.035^{*}$ \\
\hline Depression & -0.36 & 0.99 & 0.63 & 0.056 & -0.87 & $-2.60 *$ & $-3.47 \#$ & $0.012^{*}$ \\
\hline Total score & -0.50 & 1.20 & 0.71 & 0.221 & -1.97 & $-4.54^{*}$ & $-6.51 \#$ & $0.005 \#$ \\
\hline \multicolumn{9}{|l|}{ B-IPQ } \\
\hline Consequences & 0.03 & 0.50 & 0.54 & 0.221 & 0.64 & -0.23 & 0.41 & 0.770 \\
\hline Timeline & -0.28 & 0.27 & -0.01 & 0.662 & -0.42 & 0.38 & -0.04 & 0.712 \\
\hline Personal control & -0.46 & -0.22 & -0.68 & 0.430 & 1.07 & 0.45 & 1.53 & 0.504 \\
\hline Treatment control & -0.11 & 0.43 & 0.32 & 0.547 & -1.69 & $3.12 *$ & 1.43 & $0.025^{*}$ \\
\hline Identity & -0.25 & 0.07 & -0.18 & 0.773 & -0.24 & 0.79 & 0.55 & 0.589 \\
\hline Coherence & -1.12 & 0.47 & -0.65 & 0.491 & -1.13 & 0.29 & -0.83 & 0.142 \\
\hline Emotional representations & 0.68 & 0.05 & 0.73 & 0.133 & 0.29 & 0.57 & 0.86 & 0.737 \\
\hline Concerns & -0.51 & 0.77 & 0.26 & 0.108 & -0.63 & 1.30 & 0.67 & 0.359 \\
\hline \multicolumn{9}{|l|}{ UCL } \\
\hline Active coping & -0.26 & -0.27 & -0.53 & 0.808 & 1.25 & 0.84 & 2.09 & 0.628 \\
\hline Seeking distraction & 0.99 & -0.16 & 0.83 & 0.348 & -0.88 & 2.48 & 1.60 & 0.191 \\
\hline Avoiding & 0.31 & 0.27 & 0.58 & 0.711 & 0.35 & 0.36 & 0.71 & 0.834 \\
\hline
\end{tabular}


Table 4 Continued.

\begin{tabular}{l} 
\\
\\
\hline Seeking social support \\
Passive coping \\
Expression of emotions \\
Fostering reassuring thoughts \\
GSE \\
Total score \\
IPA \\
Autonomy indoors \\
Family role \\
Autonomy outdoors \\
Social life and relationships \\
Work and education \\
CushingQoL \\
Psychosocial issues \\
Physical problems \\
Total score \\
AcroQoL \\
Physical score \\
Psychological-appearance \\
Psychological-personal relations \\
Total score \\
\hline
\end{tabular}

\begin{tabular}{|c|c|c|}
\hline \multicolumn{3}{|c|}{ Patients $(n=174)$} \\
\hline $\begin{array}{c}\triangle \text { PPEP- } \\
\text { control } \\
\text { T0-T1 }\end{array}$ & $\begin{array}{c}\triangle \text { PPEP- } \\
\text { control } \\
\text { T1-T2 }\end{array}$ & $\begin{array}{c}\triangle \text { PPEP- } \\
\text { control } \\
\text { T0-T2 }\end{array}$ \\
\hline-0.48 & 0.21 & -0.26 \\
\hline 0.41 & 0.64 & 1.05 \\
\hline 0.07 & -0.07 & 0.00 \\
\hline-0.16 & -0.04 & -0.20 \\
\hline $1.35 *$ & 0.39 & $1.74 *$ \\
\hline-0.01 & .12 & 0.11 \\
\hline-0.00 & -0.03 & -0.03 \\
\hline-0.08 & 0.01 & -0.07 \\
\hline 0.06 & -0.34 & 0.02 \\
\hline-0.07 & 0.26 & 0.19 \\
\hline 4.16 & -4.36 & -0.20 \\
\hline 3.68 & -1.06 & 2.63 \\
\hline 3.83 & -4.07 & -0.24 \\
\hline 3.32 & -15.64 & -12.31 \\
\hline-6.72 & -2.05 & -8.77 \\
\hline 5.65 & -18.17 & -12.52 \\
\hline 1.25 & -12.16 & -10.91 \\
\hline
\end{tabular}

\begin{tabular}{l}
\hline \\
\hline $\begin{array}{c}P \text { value } \\
\text { group } \times \\
\text { time }\end{array}$ \\
\hline 0.544 \\
0.281 \\
0.944 \\
0.907 \\
\\
$0.020 *$ \\
\\
0.247 \\
0.956 \\
0.657 \\
0.694 \\
0.869 \\
\\
0.337 \\
0.666 \\
0.304 \\
\\
0.101 \\
0.504 \\
0.124 \\
0.149 \\
\hline
\end{tabular}

\begin{tabular}{c}
\multicolumn{2}{c}{ Pa } \\
\hline$\triangle$ PPEP- \\
control \\
T0-T1 \\
\hline 0.71 \\
-0.96 \\
-0.35 \\
-1.31 \\
\\
0.45 \\
\\
NA \\
NA \\
NA \\
NA \\
NA \\
NA \\
NA \\
NA \\
NA \\
NA \\
NA \\
NA \\
\end{tabular}

\begin{tabular}{c}
$\Delta$ PPE \\
cont \\
T1-T \\
\hline-1.6 \\
0.2 \\
0.38 \\
1.63 \\
-0.7 \\
\\
NA \\
NA \\
NA \\
NA \\
NA \\
NA \\
NA \\
NA \\
NA \\
NA \\
NA \\
NA
\end{tabular}

\section{$=63)$}

$\triangle P P$

$\triangle$ PPEP-

control T0-T2

$-0.92$

$-0.76$

0.03

0.33

$-0.32$

NA

NA

NA

NA

NA

NA

NA

NA

NA

NA

NA

NA

NA

NA

NA

NA

NA

NA
$P$ value

group $x$

time

0.501

0.503

0.771

0.111

0.830

NA

NA

NA

NA

NA

NA

NA

NA

NA

NA

NA

NA

${ }^{*} P<.05,{ }^{\#} P<.005 .{ }^{\dagger}$ Only patients with Cushing's disease; ${ }^{\ddagger}$ Only patients with acromegaly. LBNQ-Pituitary, Leiden Bother and Needs Questionnaire for pituitary diseases; EQ-5D, EuroQoL-5D; SF-36, Short Form 36; MFI-20, Multidimensional Fatigue Inventory; HADS, Hospital Anxiety and Depression Scale; B-IPQ, Brief IIIness Perception Questionnaire; UCL, Utrecht Coping List; GSE, General Self-Efficacy Scale; IPA, Impact on Participation and Autonomy questionnaire. $P$ value group $x$ time: significance of the interaction i.e., PPEP-Pituitary vs. control group $x$ time point (i.e., baseline (T0), directly after PPEP-Pituitary (T1), 6 months follow-up (T2)).

T2 relative to $\mathrm{T} 1$ (difference $-4.54, P=0.034$ ) compared to controls.

\section{Post hoc analysis: Clinical sample}

All findings from the ITT analyses were also observed in the clinical sample analysis (Supplementary Data 4). However, some new findings were observed. Patients in PPEPPituitary reported a higher need for support for coping with negative illness perceptions (LBNQ-Pituitary) than those in controls (difference $7.88, P=0.018$ ) at $\mathrm{T} 2$ relative to $\mathrm{T} 1$, but this increase at $\mathrm{T} 2$ was not significantly different from baseline (difference 3.14, $P=0.422$ ). Furthermore, PPEPPituitary reported a higher need for support for physical and cognitive problems (LBNQ-Pituitary) at T2 relative to T1 (difference 7.01, $P=0.023$ ), which was also significantly different from baseline (difference 7.43, $P=0.036$ ). PPEPPituitary reported more depressive symptoms (HADS) (difference $1.17, P=0.008$ ) at $\mathrm{T} 2$ relative to $\mathrm{T} 1$, but this increase at $\mathrm{T} 2$ was not significantly different from baseline (difference 0.67, $P=0.191$ ). Partners in PPEP-Pituitary reported better social functioning (SF-36) at T2 relative to T1 (difference 19.70, $P=0.023$ ) compared to controls, which was also significantly different from baseline (difference 22.30, $P=0.012$ ).

\section{Patient and partner evaluation}

Of the patients who followed at least 6 sessions i.e., the clinical sample $(n=60), 55$ patients filled out the evaluation form (92\%). Ninety-five per cent of the patients agreed that the exchange of experiences within the group was helpful, and over half of the patients (53\%) reported a better understanding of the psychological effects of their disease. Two-thirds of the patients (67\%) reported their expectations were fulfilled, and $84 \%$ would recommend the programme to other patients. All partners who followed at least 6 sessions ( $n=13)$ filled out the evaluation form. All partners agreed that the exchange of experiences was helpful; two-thirds of the partners (62\%) reported a better understanding of the psychological effects of the disease. In $54 \%$ of the partners, their expectations were fulfilled, and $77 \%$ would recommend the programme to other partners. 


\section{Discussion}

PPEP-Pituitary resulted in enhanced self-efficacy in patients which persisted after the 6-month follow-up. Perceived bother by mood problems decreased directly after PPEP-Pituitary, but returned to baseline level after 6-month follow-up. Partners reported more vitality immediately after PPEP-Pituitary, which was still present after 6 months. Partners also reported less anxiety and depressive symptoms after 6 months. Furthermore, mood improved after each session (except for session 1) in patients and after the last three sessions in partners.

Similar to the results of the SMI described by Haugland et al. (10), PPEP-Pituitary enhanced self-efficacy in patients. The term self-efficacy is described in the 'Social Cognitive Theory' of Bandura (40) and defined as the person's beliefs in his or her own capabilities to perform a certain action, in a certain environment. Following this model, behaviour is directly influenced by goals and self-efficacy expectations. In line with this model, several studies demonstrated that self-efficacy influences self-management behaviour $(41,42)$, as well as SMIs improving self-efficacy in patients with chronic disease $(43,44)$. For instance, Steed et al. evaluated a SMI which was based on the Social Cognitive Theory and demonstrated a positive effect on diabetes selfmanagement behaviour, i.e., diabetes-specific diet, exercise and blood glucose monitoring (45). Following Bandura, self-efficacy can be increased and behaviour change is enhanced by four components: 1) mastery, which refers to the direct experience of success in performing a certain behaviour; 2) vicarious experience, which refers to modelling gained by successful behaviour of a person with whom one identifies (e.g., person with the same illness); 3) social persuasion e.g., encouragement from health professionals or members of the self-management group; and 4) reducing feelings of stress and altering negative emotional tendencies, since this may lead to reducing misinterpretations of physical symptoms or one's physical state (46). All four components were used in PPEP-Pituitary.

The results of the ITT analysis were further confirmed by the analyses including only participants that followed at least six sessions (i.e., the clinical sample). In the clinical sample analysis as well as in the ITT analysis, we observed that depressive symptoms and bother by mood problems increased during 6-month follow-up after PPEPPituitary, although not different from baseline levels. Furthermore, the clinical sample analysis complemented the ITT results by observations that patients reported a higher need for support for coping with negative illness perceptions and physical and cognitive problems. This finding might be explained by the fact that patients in PPEP-Pituitary learned skills to concretize/verbalize their healthcare needs, but also suggests that it might be useful to implement one or two additional refreshing/booster sessions during follow-up e.g., after 6 months or even over 12 months. On the other hand, partners reported an increase in social functioning 6 months after PPEPPituitary. This seems to indicate that partners needed time to implement the newly learned skills in their daily lives. It could also be that aspects of QoL improved in partners as a result of the improvement in self-efficacy in their ill partners.

In the present study, we did not observe any effects in patients on QoL, illness perceptions, coping, and autonomy and participation in different life domains. It should, however, be noted that there was a relatively long duration since diagnosis (i.e., PPEP-Pituitary: 12 years, control group: 13 years). It is conceivable that during this long period of living with the disease, patients and partners adapted to the consequences of the disease and/ or already had received appropriate support, which may have limited the beneficial effects of our programme in improving psychosocial aspects. It should also be realized that although some aspects did not change during the time of the study, it could be that due to the learned psychosocial skills, patients and partners became more resilient to develop psychosocial morbidity, and future research into this area is warranted.

Due to the explorative nature of the present study, a large number of outcome parameters was used which could have led to a higher chance of type I error. After the post hoc Bonferroni correction, the effect on mood problems in patients and the effect on anxiety and depressive symptoms could still be observed. Furthermore, the large number of outcome parameters could also have influenced the response rate of the participants, considering the duration of filling out the questionnaires. In addition, it should be acknowledged that selfmanagement is, by definition, largely implemented by the participants themselves with limited external supervision. For instance, it is not known how often participants practised the learned skills at home. This information could have provided additional insight into the effects of the program, and should therefore be taken into account in future research by, for instance, asking participants to keep up a diary. Another limitation related to research 
in SMIs is that they largely rely on self-report measures. The measures used in this study were all validated, but probably not equal to direct observation.

A strength of the present study is the inclusion of both patients and partners, and the relatively large sample size. In addition, the variability in included centres as well as in trainers $(n=9)$ (i.e. psychologist, medical social workers) increases the validity of the effect of PPEPPituitary. For future implementation of the programme in (other) medical centres, PPEP-Pituitary can be provided by psychologist and social workers, but also by other healthcare professionals such as endocrine nurses as long as they are trained in the principles of PPEP-Pituitary and have an appropriate level of knowledge about pituitary disease. Sixty-seven per cent of the patient reported that their expectation about PPEP-Pituitary were met. From the notes written on the evaluation forms it became apparent that patients would have liked more (practical, medical) information about their disease (i.e. bodily changes due to disease, medication, side-effects). Therefore, we are considering the invitation of an endocrine nurse to the first session to provide (practical) information about the disease. For future implementation of PPEP-Pituitary, it is very difficult to form separate groups per disease (i.e., CD, acromegaly, NFA/PRL), considering the low incidence of pituitary adenomas. Therefore, we postulate that groups can be formed with patients with different pituitary diseases. This seems to be suitable considering the overlapping symptoms (i.e. hypopituitarism, fatigue), but on the other hand, it can be imagined that for a patient it can be helpful to have at least one other person in the group with the same disease. Future implementation of PPEP-Pituitary groups of patients with different pituitary diseases can be formed, but with taking into account the distribution of diseases per group. Furthermore, a question that needs to be further clarified in future research is determining the best moment to offer PPEP-Pituitary during the disease process. We believe that directly after biomedical treatment is not the right moment, because patients need their time and energy to recover from treatment, but also because patients will not have a clear idea about the psychosocial consequences of the disease, making it difficult to work on during PPEP-Pituitary. On the other hand, when the programme is offered years after biomedical treatment, patient may have learned to cope with the consequences and/or they had to search for psychological care by themselves. Therefore, we postulated that the ideal moment to offer the programme will be between 6 and 12 months after biochemical remission. It is speculated that offering the programme at that time might lead to less healthcare consumption. Therefore, for future research, it would be interesting to assess the effects of PPEP-Pituitary in a clinical setting that also includes patients that have recently obtained a stable medical situation.

In conclusion, this first study about the effects of PPEP-Pituitary in a large cohort of patients with, on average, a relatively long duration since diagnosis demonstrated that PPEP-Pituitary enhances self-efficacy in patients, and their partners report better QoL in the long term. We postulate that implementing PPEP-Pituitary in clinical care will (at least partly) meet the current unmet needs regarding psychosocial care in patients with pituitary disease and their partners. For the implementation of PPEP-Pituitary, we are currently evaluating the approach to schedule one or two additional refreshing/booster sessions after 6 months or 12 months. Future research will need to focus on the implementation of this programme into clinical care trajectories.

\section{Supplementary data}

This is linked to the online version of the paper at http://dx.doi.org/10.1530/ EJE-16-1015.

\section{Funding}

This research was funded by an unrestricted educational grant from Ipsen.

\section{Acknowledgements}

The authors thank all patients and their partners for their participation. The PPEP-Pituitary trainers from the department of social work of the LUMC N Fonville, R Garritsen, J Lourier, R Manshanden, S Muntz, M van der Plas-Donkers and PPEP-Pituitary trainers M Schenk, and M Vreugdenhil. Furthermore, they thank R Wolterbeek of the Department of Medical Statistics and Bioinformatics of the LUMC for statistical advice.

\section{References}

1 van der Klaauw AA, Kars M, Biermasz NR, Roelfsema F, Dekkers OM, Corssmit EP, van Aken MO, Havekes B, Pereira AM, Pijl H et al. Disease-specific impairments in quality of life during longterm follow-up of patients with different pituitary adenomas. Clinical Endocrinology 200869 775-784. (doi:10.1111/j.13652265.2008.03288.x)

2 Andela CD, Scharloo M, Pereira AM, Kaptein AA \& Biermasz NR. Quality of life (QoL) impairments in patients with a pituitary adenoma: a systematic review of QoL studies. Pituitary 201518 752-776. (doi:10.1007/s11102-015-0636-7)

3 Andela CD, Niemeijer ND, Scharloo M, Tiemensma J, Kanagasabapathy S, Pereira AM, Kamminga NG, Kaptein AA \& Biermasz NR. Towards a better quality of life (QoL) for patients with pituitary diseases: results from a focus group study exploring QoL. Pituitary 201418 86-100. (doi:10.1007/s11102-014-0561-1)

4 Dunning T \& Alford F. Pituitary disease: perspectives of patients and partners. Journal of Nursing and Healthcare of Chronic Illness $2009 \mathbf{1}$ 139-146. (doi:10.1111/j.1752-9824.2009.01014.x) 
5 Newman S, Steed L \& Mulligan K. Chronic Physical Illness: SelfManagement and Behavioural Interventions. 1 edn. Berkshire: McGrawHill, 2009

6 Barlow J, Wright C, Sheasby J, Turner A \& Hainsworth J. Selfmanagement approaches for people with chronic conditions: a review. Patient Education and Counseling 200248 177-187. (doi:10.1016/S0738-3991(02)00032-0)

7 Martire LM, Schulz R, Helgeson VS, Small BJ \& Saghafi EM. Review and meta-analysis of couple-oriented interventions for chronic illness. Annals of Behavioral Medicine 201040 325-342. (doi:10.1007/ s12160-010-9216-2)

8 Newman S, Steed L \& Mulligan K. Self-management interventions for chronic illness. Lancet 2004364 1523-1537. (doi:10.1016/S01406736(04)17277-2)

9 Martinez-Momblan MA, Gomez C, Santos A, Porta N, Esteve J, Ubeda I, Halperin I, Campillo B, Guillaumet M, Webb SM et al. A specific nursing educational program in patients with Cushing's syndrome. Endocrine 201553 199-209. (doi:10.1007/s12020-015-0737-0)

10 Haugland T, Veenstra M, Vatn MH \& Wahl AK. Improvement in stress, general self-efficacy, and health related quality of life following patient education for patients with neuroendocrine tumors: a pilot study. Nursing Research and Practice 2013 1-9. (doi:10.1155/2013/695820)

11 Macht M, Gerlich C, Ellgring H, Schradi M, Rusinol AB, Crespo M, Prats A, Viemero V, Lankinen A, Bitti PE et al. Patient education in Parkinson's disease: Formative evaluation of a standardized programme in seven European countries. Patient Education and Counseling 200765 245-252. (doi:10.1016/j.pec.2006.08.005)

12 A'Campo LE, Spliethoff-Kamminga NG, Macht M \& Roos RA. Caregiver education in Parkinson's disease: formative evaluation of a standardized program in seven European countries. Quality of Life Research 201019 55-64. (doi:10.1007/s11136-009-9559-y)

13 A'Campo LE, Wekking EM, Spliethoff-Kamminga NG, Le CS $\&$ Roos RA. The benefits of a standardized patient education program for patients with Parkinson's disease and their caregivers. Parkinsonism \& Related Disorders 201016 89-95. (doi:10.1016/j. parkreldis.2009.07.009)

14 A'Campo LE, Spliethoff-Kamminga NG \& Roos RA. An evaluation of the patient education programme for Parkinson's disease in clinical practice. International Journal of Clinical Practice 201165 1173-1179. (doi:10.1111/j.1742-1241.2011.02765.x)

15 A'Campo LE, Wekking EM, Spliethoff-Kamminga NG, Stijnen T $\&$ Roos RA. Treatment effect modifiers for the patient education programme for Parkinson's disease. International Journal of Clinical Practice 201266 77-83. (doi:10.1111/j.1742-1241.2011.02791.x)

16 A'Campo LE, Spliethoff-Kamminga NG \& Roos RA. The patient education program for Huntington's disease (PEP-HD). Journal of Huntington's Disease 20121 47-56. (doi:10.3233/JHD-2012-120002)

17 A'Campo LE. A patient and caregiver education program (Doctoral dissertation). Retrieved from https://openaccess.leidenuniv.nl/ handle/1887/19043. 2012 151-168.

18 Kamminga $\mathrm{N} \&$ Bustraan J. PPEP4All: Zelfmanagementprogramma voor chronisch zieken en hun partner - Patiëntenwerkboek \& Partnerwerkboek (PPEP4ALL: Selfmanagement programme for people with chronic disease and their caregivers- Manual for the patient and manual for the caregiver). Amsterdam: Uitgeverij Boom, 2014.

19 Weigold A, Weigold IK \& Russell EJ. Examination of the equivalence of self-report survey-based paper-and-pencil and internet data collection methods. Psychological Methods 201318 53-70. (doi:10.1037/a0031607)

20 Schwarzer R \& Jerusalem M. Generalized Self-efficacy scale. In Measures in Health Psychology: A User's Portfolio. Causal and Control Beliefs. pp 35-37. Eds J Weinman, S Wright \& M Johnston. Windsor, England: NFER-NELSON, 1995.

21 Scholz U, Gutiérrez-Dona B, Sud S \& Schwarzer R. Is general selfefficacy a universal construct? Psychometric findings from 25 countries. European Journal of Psychological Assessment 201518 242-251. (doi:10.1027//1015-5759.18.3.242)

22 Andela CD, Scharloo M, Ramondt S, Tiemensma J, Husson O, Llahana S, Pereira AM, Kaptein AA, Kamminga NG \& Biermasz NR. The development and validation of the Leiden Bother and needs questionnaire for patients with pituitary disease: the LBNQ-Pituitary. Pituitary 201619 293-302. (doi:10.1007/s11102-016-0707-4)

23 Moss-Morris R, Weinman J, Petrie K, Horne R, Cameron L \& Buick D. The revised illness perception questionnaire (IPQ-R). Psychology \& Health 2002 17 1-16.

24 Scheurs P, van de Willige G, Brosschot J, Tellegen B \& Graus G. De Utrechtse coping lijst: UCL. Lisse, The Netherlands: Swets en Zeitlinger b.v., 1993.

25 Cardol M, de Haan RJ, de Jong BA, van den Bos GA \& de Groot IJ. Psychometric properties of the Impact on Participation and Autonomy Questionnaire. Archives of Physical Medicine and Rehabilitation 200182 210-216. (doi:10.1053/apmr.2001.18218)

26 EuroQoL group. EuroQol-a new facility for the measurement of health-related quality of life. The EuroQol Group. Health Policy 1990 16 199-208. (doi:10.1016/0168-8510(90)90421-9)

27 Brazier JE, Harper R, Jones NM, O'Cathain A, Thomas KJ, Usherwood $\mathrm{T} \&$ Westlake L. Validating the SF-36 health survey questionnaire: new outcome measure for primary care. British Medical Journal 1992 305 160-164. (doi:10.1136/bmj.305.6846.160)

28 Ware JE, Jr. \& Sherbourne CD. The MOS 36-item short-form health survey (SF-36). I. Conceptual framework and item selection. Medical Care 199230 473-483. (doi:10.1097/00005650-199206000-00002)

29 Smets EM, Garssen B, Bonke B \& De Haes JC. The Multidimensional Fatigue Inventory (MFI) psychometric qualities of an instrument to assess fatigue. Journal of Psychosomatic Research 199539 315-325. (doi:10.1016/0022-3999(94)00125-O)

30 Snaith RP \& Zigmond AS. The hospital anxiety and depression scale. British Medical Journal 1986292 344. (doi:10.1136/bmj.292.6516.344)

31 Spinhoven P, Ormel J, Sloekers PP, Kempen GI, Speckens AE \& van Hemert AM. A validation study of the Hospital Anxiety and Depression Scale (HADS) in different groups of Dutch subjects. Psychological Medicine 199727 363-370. (doi:10.1017/ S0033291796004382)

32 Badia X, Webb SM, Prieto L \& Lara N. Acromegaly quality of life questionnaire (AcroQoL). Health and Quality of Life Outcomes 20042 13-18. (doi:10.1186/1477-7525-2-13)

33 Webb SM, Badia X \& Surinach NL. Validity and clinical applicability of the acromegaly quality of life questionnaire, AcroQoL: a 6-month prospective study. European Journal of Endocrinology 2006155 269-277. (doi:10.1530/eje.1.02214)

34 Webb SM, Prieto L, Badia X, Albareda M, Catala M, Gaztambide S, Lucas T, Paramo C, Pico A, Lucas A et al. Acromegaly quality of life questionnaire (ACROQOL) a new health-related quality of life questionnaire for patients with acromegaly: development and psychometric properties. Clinical Endocrinology 200257 251-258. (doi:10.1046/j.1365-2265.2002.01597.x)

35 Webb SM, Badia X, Barahona MJ, Colao A, Strasburger CJ, Tabarin A, van Aken MO, Pivonello R, Stalla G, Lamberts SW et al. Evaluation of health-related quality of life in patients with Cushing's syndrome with a new questionnaire. European Journal of Endocrinology 2008158 623-630. (doi:10.1530/EJE-07-0762)

36 Santos A, Resmini E, Martinez-Momblan MA, Crespo I, Valassi E, Roset M, Badia X \& Webb SM. Psychometric performance of the CushingQoL questionnaire in conditions of real clinical practice. European Journal of Endocrinology 2012167 337-342. (doi:10.1530/EJE12-0325)

37 Tiemensma J, Depaoli S \& Felt JM. Using subscales when scoring the Cushing's quality of life questionnaire. European Journal of Endocrinology 2016174 33-40. (doi:10.1530/EJE-15-0640)

38 Krueger C \& Tian L. A comparison of the general linear mixed model and repeated measures ANOVA using a dataset with multiple 
missing data points. Biological Research for Nursing 20046 151-157. (doi:10.1177/1099800404267682)

39 Gupta SK. Intention-to-treat concept: a review. Perspectives in Clinical Research 20112 109-112. (doi:10.4103/2229-3485.83221)

40 Bandura A. Social Foundations of Thought and Action: A Social Cognitive Theory. Englewood Cliffs, New Jersey: Prentice-Hall, Inc., 1986.

41 Abubakari AR, Cousins R, Thomas C, Sharma D \& Naderali EK. Sociodemographic and clinical predictors of self-management among people with poorly controlled type 1 and type 2 diabetes: the role of illness perceptions and self-efficacy. Journal of Diabetes Research 2016 2016 1-12. (doi:10.1155/2016/6708164)

42 Wilski M \& Tasiemski T. Illness perception, treatment beliefs, self-esteem, and self-efficacy as correlates of self-management in multiple sclerosis. Acta Neurologica Scandinavica 2015133 338-345. (doi:10.1111/ane.12465)
43 Hemmati MM \& Raiesi Z. Effect of a self-management and follow-up program on self-efficacy in patients with multiple sclerosis: a randomized clinical trial. Nursing and Midwifery Studies 20143 e25661. (doi:10.17795/nmsjournal25661)

44 Damush TM, Kroenke K, Bair MJ, Wu J, Tu W, Krebs EE \& Poleshuck E. Pain self-management training increases self-efficacy, selfmanagement behaviours and pain and depression outcomes. European Journal of Pain 201620 1070-1078. (doi:10.1002/ejp.830)

45 Steed L, Lankester J, Barnard M, Earle K, Hurel S \& Newman S. Evaluation of the UCL diabetes self-management programme (UCLDSMP): a randomized controlled trial. Journal of Health Psychology 200510 261-276. (doi:10.1177/1359105305049775)

46 Bandura A. Health promotion from the perspective of social cognitive theory. Psychology and Health 199813 623-649. (doi:10.1080/08870449808407422)

Received 12 December 2016

Revised version received 24 March 2017

Accepted 19 April 2017 